\title{
MicroRNA-503 inhibits the proliferation and invasion of breast cancer cells via targeting insulin-like growth factor 1 receptor
}

\author{
JINGWANG YAN $^{1}$, YONGHUAN XU ${ }^{2}$, HAIPENG WANG ${ }^{1}$, TAIPING DU ${ }^{1}$ and HAO CHEN ${ }^{1}$ \\ ${ }^{1}$ Department of General Surgery, Xinxiang Center Hospital, Xinxiang, Henan 453000; \\ ${ }^{2}$ Department of Oncology, People's Hospital of Xixia County, Nanyang, Henan 474550, P.R. China
}

Received April 5, 2016; Accepted March 7, 2017

DOI: $10.3892 / \mathrm{mmr} .2017 .6816$

\begin{abstract}
MicroRNAs (miRs), a class of non-coding RNAs that are 18-25 nucleotides in length, serve as key regulators in the development and progression of human cancers. Previously, miR-503 has been implicated in breast cancer. However, the underlying mechanism of miR-503 in regulating the proliferation and invasion of breast cancer cells remains largely unknown. In the present study, reverse transcription-quantitative polymerase chain reaction analysis indicated that the expression of miR-503 was significantly reduced in breast cancer tissues compared with their matched adjacent normal tissues. Furthermore, miR-503 expression levels were markedly reduced in T2-T4 stage breast cancer, compared with T1 stage. Insulin-like growth factor 1 receptor (IGF-1R) was further identified as a novel target of miR-503. Overexpression of miR-503 significantly suppressed the protein expression levels of IGF-1R. Furthermore, it inhibited the proliferation and invasion of human breast cancer MCF-7 cells, as assessed by MTT and Transwell assays, respectively. However, restoration of IGF-1R expression markedly ameliorated the suppressive effects of miR-503 overexpression on MCF-7 cell proliferation and invasion, indicating that miR-503 inhibits breast cancer cell proliferation and inyasion at least partially via directly targeting IGF-1R. Furthermore, the mRNA and protein expression levels of IGF-1R were demonstrated to be significantly increased in breast cancer tissues compared with their matched adjacent normal tissues. In addition, IGF-1R mRNA expression levels were reversely correlated with miR-503 expression levels in breast tumors, suggesting that the upregulation of IGF-1R may be due to downregulation of miR-503 in breast cancer. In conclusion, the present study expanded the understanding of the regulatory mechanism of
\end{abstract}

Correspondence to: Dr Jingwang Yan, Department of General Surgery, Xinxiang Center Hospital, 56 Jinsui Road, Xinxiang, Henan 453000, P.R. China

E-mail: xinxiangyjw@sina.com

Key words: breast cancer, microRNA-503, insulin-like growth factor 1 receptor, proliferation, invasion
miR-503 in breast cancer, and implicates the miR-503/IGF-1R axis as a potential therapeutic target for breast cancer.

\section{Instruction}

Breast cancer, the second most common type of cancer worldwide, is the leading cause of cancer mortality in females, accounting for $\sim 15 \%$ of all cancer deaths (1). Deregulation of various oncogenes and tumor suppressors have been demonstrated to serve key roles in the development and progression of breast cancer $(2,3)$. Therefore, it is urgently required to investigate the underlying molecular mechanisms to help develop more effective therapeutic strategies for breast cancer patients.

Growth and metastasis of breast cancer are closely associated with deregulation, mutation and epigenetic mechanisms of certain genes, including microRNAs (miRs) (2-5). miRs are a class of non-coding RNAs that are 18-25 nucleotides in length, and serve as regulators of gene expression via directly binding to the 3'-untranslational region (UTR) of their target mRNAs, which causes mRNA degradation or translation inhibition $(6,7)$. Through mediation of their targets, miRs serve important regulatory roles in a variety of biological processes, including cell survival, proliferation, differentiation, cell cycle progression, migration and invasion $(7,8)$. In recent years, evidence on the roles of miRs in breast cancer have widely been reported. Among these miRs, miR-503 was originally reported to be involved in inflammatory breast cancer, a rare but very aggressive form of breast cancer with a particular phenotype (9). Long et al (10) further demonstrated that miR-503 was markedly downregulated in breast cancer, and overexpression of miR-503 suppressed the proliferation of breast cancer cells through inducing G0/G1 cell cycle arrest by targeting cyclin D1, suggesting that miR-503 serves as a tumor suppressor in breast cancer. Furthermore, Polioudakis et al (11) suggested that miR-503 was a negative regulator of proliferation in primary breast cancer cells via targeting DDHD domain containing 2. However, the molecular mechanism of miR-503 in the regulation of breast cancer growth and metastasis remains largely unknown.

Insulin-like growth factor 1 receptor (IGF-1R), a member of the IGF receptor family, directly binds to IGF and activates the downstream signaling pathway, and is involved in the malignant transformation by promoting cell survival and 
inhibiting cell apoptosis (12-14). It has been demonstrated to be significantly upregulated in breast cancer $(15,16)$. Furthermore, IGF-1R has been demonstrated to promote the growth and metastasis of breast cancer (17). IGF-1R has been developed into an important therapeutic target for breast cancer $(18,19)$. Lentivirus-mediated short-hairpin RNA targeting IGF-1R has been demonstrated to inhibit growth and lymphangiogenesis in breast cancer (19). However, the regulatory mechanism of IGF-1R expression remains largely unclear; investigating this may facilitate the development of effective therapeutic strategies for breast cancer treatment.

Therefore, the present study aimed to investigate the underlying molecular mechanism of miR-503 in regulating the proliferation and invasion of breast cancer cells.

\section{Materials and methods}

Tissue specimen collection. The present study was approved by the Ethical Committee of Xinxiang Center Hospital (Xinxiang, China). A total of 23 breast cancer tissues and 23 matched adjacent normal tissues were obtained from June 2013 to March 2014 at the Department of Gynecology and Obstetrics, Xinxiang Center Hospital. All female patients were diagnosed as having primary breast cancer and ranged in age from 33 to 67 years, with a mean age of 51.5 years. Patients received no chemotherapy or radiotherapy prior to surgical resection. Written consents were obtained from the patients in this study. All tissues were immediately snap-frozen in liquid nitrogen after surgical removal, and stored at $-80^{\circ} \mathrm{C}$ until further use.

Immunohistochemical staining. The expression of IGF1R was evaluated using immunohistochemical staining. Sections (4- $\mu \mathrm{m}$ thick) were deparaffinized and subjected to heat-induced antigen retrieval using citrate buffer for 22 min using a microwave oven. Then the sections were incubated with a primary antibody against IGF1R (1:100; cat. no. ab39675, Abcam, Cambridge, MA, USA) at $4^{\circ} \mathrm{C}$ for $24 \mathrm{~h}$. Subsequently, the sections were incubated with HRP conjugated goat-anti-rabbit IgG (1:5,000; cat. no. ab6721, Abcam) for $60 \mathrm{~min}$ at room temperature. The reaction was developed using diaminobenzidine (DAB) and counterstained with hematoxylin, and observed under a CX23 microscope (Olympus Corporation, Tokyo, Japan).

Cell culture. The MCF-7 human breast cancer cell line was purchased from the Cell Bank of Chinese Academy of Sciences (Shanghai, China). Cells were cultured in Dulbecco's modified Eagle's medium (DMEM; Thermo Fisher Scientific, Inc., Waltham, MA, USA) supplemented with $10 \%$ fetal bovine serum (FBS, Thermo Fisher Scientific, Inc.) at $37^{\circ} \mathrm{C}$ in a humidified incubator containing $5 \% \mathrm{CO}_{2}$.

Reverse transcription quantitative polymerase chain reaction (RT-qPCR). Total RNA of tissues and cells was extracted using TRIzol ${ }^{\circledR}$ Reagent (Thermo Fisher Scientific, Inc.), according to the manufacture's protocol. A total of $500 \mathrm{ng}$ RNA was converted into cDNA using a Reverse Transcription kit (Thermo Fisher Scientific, Inc.), according to the manufacture's protocol. Reverse transcription was performed at $16^{\circ} \mathrm{C}$ for $30 \mathrm{~min}$, followed by incubation at $42^{\circ} \mathrm{C}$ for $30 \mathrm{~min}$ and enzyme inactivation at $85^{\circ} \mathrm{C}$ for $5 \mathrm{~min}$. For miR-503 expression detection, a miRNA qPCR Detection kit (GeneCopoeia, Inc., Rockville, MD, USA) was then used for PCR on an ABI 7500 thermocycler system (Thermo Fisher Scientific, Inc.), according to the manufacture's protocol. For mRNA expression detection, SYBR Green qPCR Master mix (Bio-Rad Laboratories, Inc., Hercules, CA, USA) was used for PCR. The following primers were used: Forward, 5'-ATGCTGACC TCTGTTACCTCT-3' and reverse, 5'-GGCTTATTCCCC ACAATGTAGTT-3' for IGF-1R; and forward, 5'-CCACTA GGCGCTCACTGTT-3' and reverse, 5'-TGGAATTTGCCA TGGGTGGA-3' for GAPDH. The following thermocycling conditions were used: initial denaturation at $95^{\circ} \mathrm{C}$ for $5 \mathrm{~min}$ followed by 40 cycles of denaturation at $95^{\circ} \mathrm{C}$ for $15 \mathrm{sec}$, and annealing/elongation at $60^{\circ} \mathrm{C}$ for $30 \mathrm{sec}$. The U6 gene served as an internal reference. This experiment was repeated for 3 times. The relative expression was analyzed by the $2^{-\Delta \Delta C q}$ method (20).

Western blot analysis. Cells were solubilized in cold radioimmunoprecipitation lysis buffer (Thermo Fisher Scientific, Inc.). The protein was extracted by centrifugation at $12,000 \mathrm{x}$ g for $20 \mathrm{~min}$ at $4^{\circ} \mathrm{C}$. The concentration of protein was determined using BCA Protein Assay kit (Pierce; Thermo Fisher Scientific, Inc.). Proteins (50 $\mu \mathrm{g} /$ lane) were separated by $10 \%$ SDS-PAGE and subsequently transferred onto a polyvinylidene difluoride membrane (Thermo Fisher Scientific, Inc.). The membrane was incubated with PBS containing $5 \%$ milk overnight at $4{ }^{\circ} \mathrm{C}$, which was then incubated with mouse anti-IGF-1R (1:200) and mouse anti-GAPDH (1:100, cat. no. ab8245, Abcam) monoclonal antibodies at room temperature for $3 \mathrm{~h}$. After three washes with PBS, the membrane was incubated with a rabbit anti-mouse secondary antibody (1:10,000, cat. no. ab6728, Abcam) at room temperature for $1 \mathrm{~h}$. Chemiluminescent detection was conducted using an Enhanced Chemiluminescence kit (Pierce; Thermo Fisher Scientific, Inc.), according to the manufacturer's protocol.

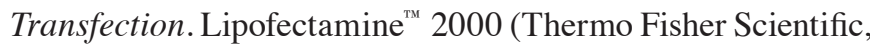
Inc.) was used to perform cell transfection, according to the manufacturer's protocol. For miR-503 and IGF-1R function analysis, MCF-7 cells were transfected with negative control miR (miR-NC), miR-503 mimic or miR-503 inhibitor, all purchased from Thermo Fisher Scientific, Inc., or co-transfected with miR-503 mimic and IGF-1R plasmid (purchased from Amspring, Changsha, China).

MTT assay. To examine cell proliferation, $2 \times 10^{4} \mathrm{MCF}-7$ cells in each group were cultured in a 96-well plate, in which $100 \mu \mathrm{l}$ DMEM containing $0.5 \mathrm{~g} / 1$ MTT (Thermo Fisher Scientific, Inc.) was added to each well. Cells were then cultured for $12,24,48$ or $72 \mathrm{~h}$. The medium was removed, and $50 \mu \mathrm{l}$ dimethyl sulfoxide (Sigma-Aldrich; Merck KGaA, Darmstadt, Germany) was added. After incubation at $37^{\circ} \mathrm{C}$ for $10 \mathrm{~min}$, the A570 of each sample was measured at a wavelength of $570 \mathrm{~nm}$ using a plate reader (TECAN Infinite M200, Tecan Group Ltd., Männedorf, Switzerland). Control cells were treated with DMSO only. 
A

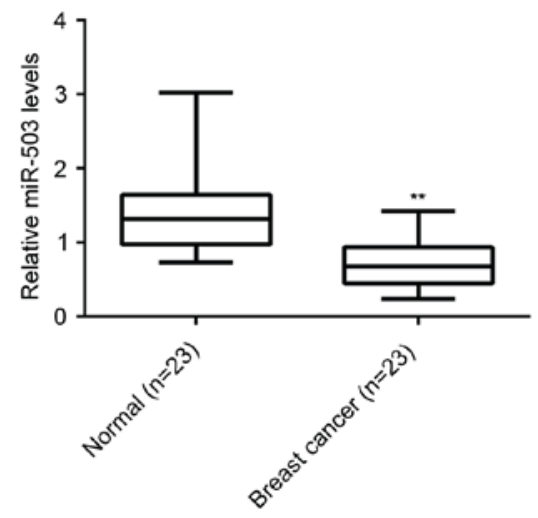

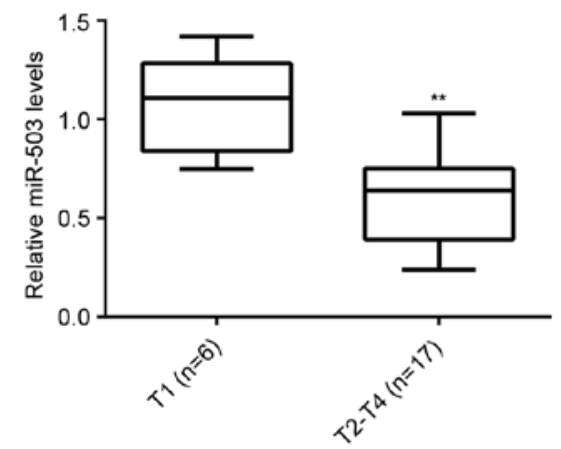

Figure 1. miR-503 is significantly downregulated in breast cancer. Reverse transcription-quantitative polymerase chain reaction was performed to examine the expression levels of miR-503 in (A) breast cancer tissues and their matched adjacent normal tissues ( ${ }^{* *} \mathrm{P}<0.01$ vs. Normal) and (B) T1 ( $\left.\mathrm{n}=6\right)$ and T2-T4 stages $\left(\mathrm{n}=17 ;{ }^{* *} \mathrm{P}<0.01\right.$ vs. T1). Data are expressed as the mean \pm standard deviation. miR, microRNA; $\mathrm{T}$, tumor.
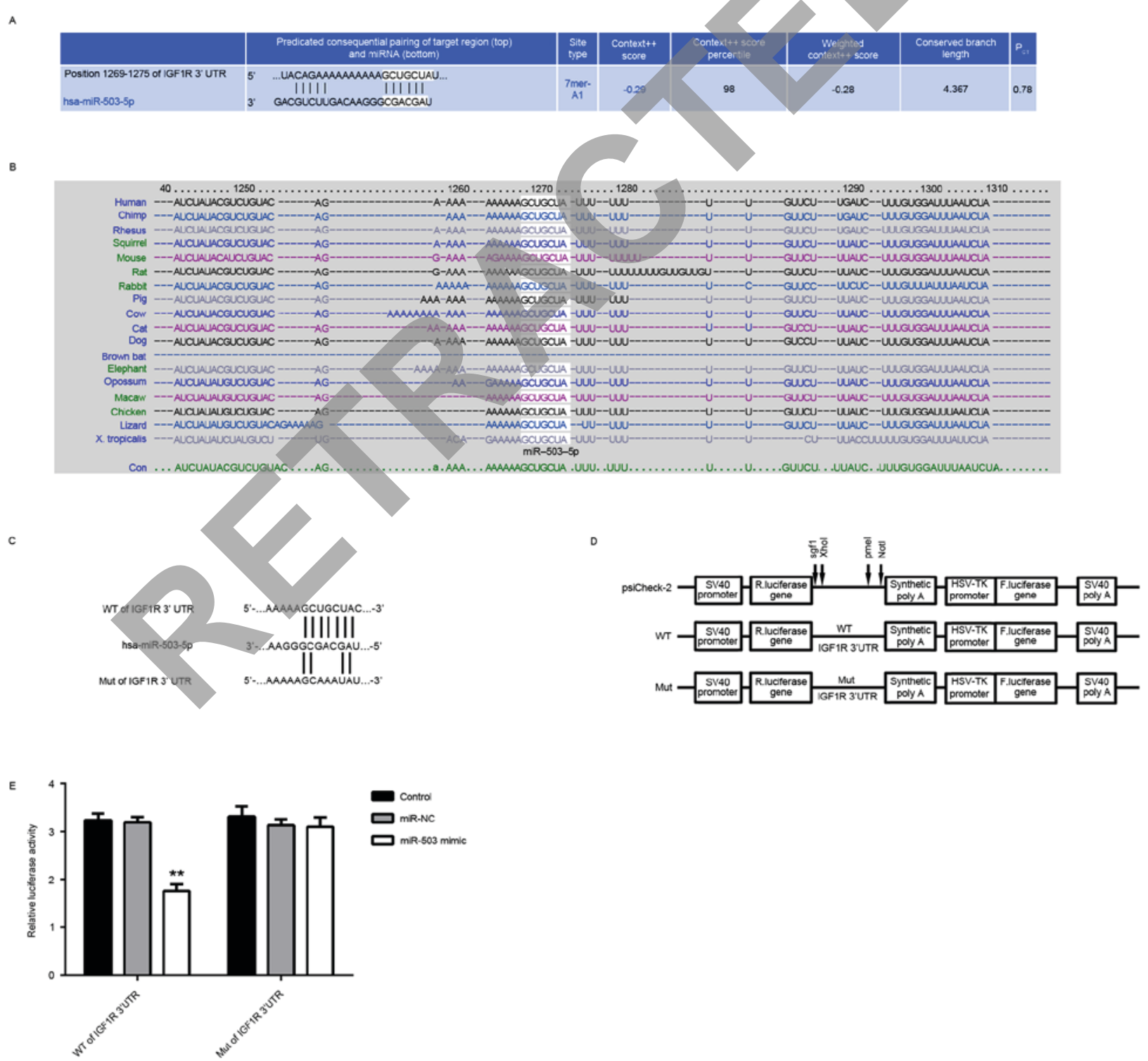

Figure 2. IGF-1R is a target gene of miR-503 in MCF-7 cells. (A) Targetscan software predicated that IGF-1R was a potential target of miR-503, and (B) this targeting relationship was evolutionally conserved. (C and D) The luciferase reporter vectors containing the WT and MUT of IGF-1R 3'-UTR were constructed (E) The luciferase activity was significantly decreased in MCF-7 cells co-transfected with the WT of IGF-1R 3'UTR reporter vector and miR-503 mimic, but demonstrated no difference in cells co-transfected with the MUT of IGF-1R 3'UTR reporter vector and miR-503 mimic, compared with the control group. ${ }^{* *} \mathrm{P}<0.01$ vs. Control. NC, negative control; miR, microRNA; MUT, mutant; WT, wild-type; UTR, untranslated region; IGF-1R, insulin-like growth factor receptor 1; R. luciferase, Renilla luciferase. 
A

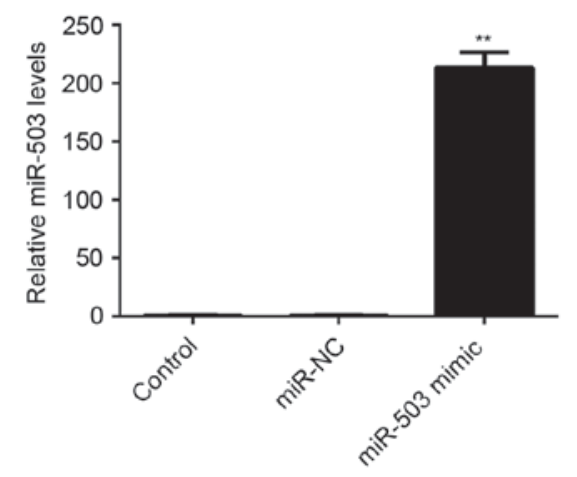

C
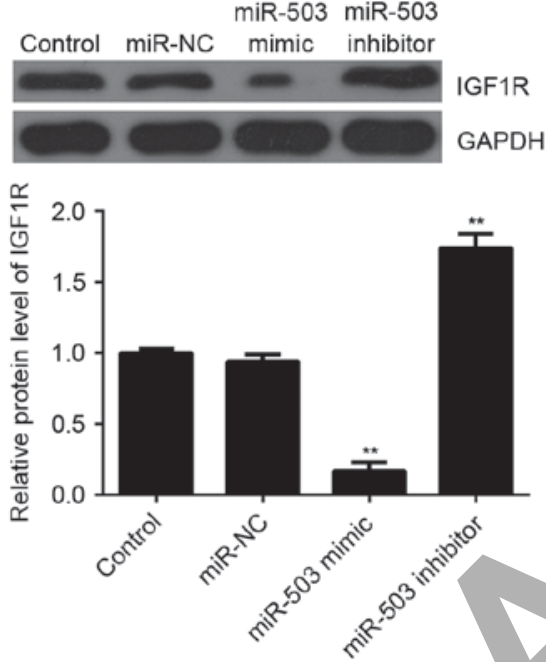

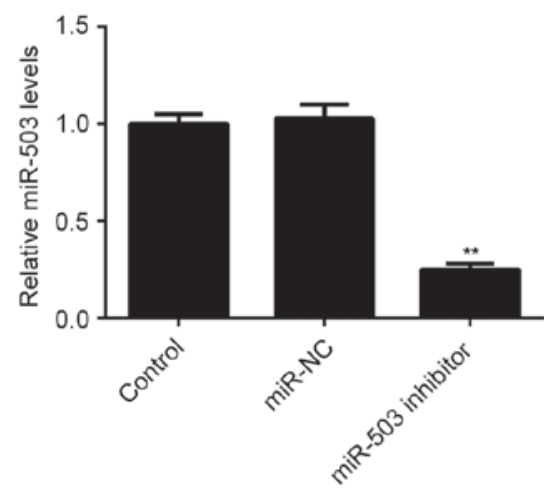

D

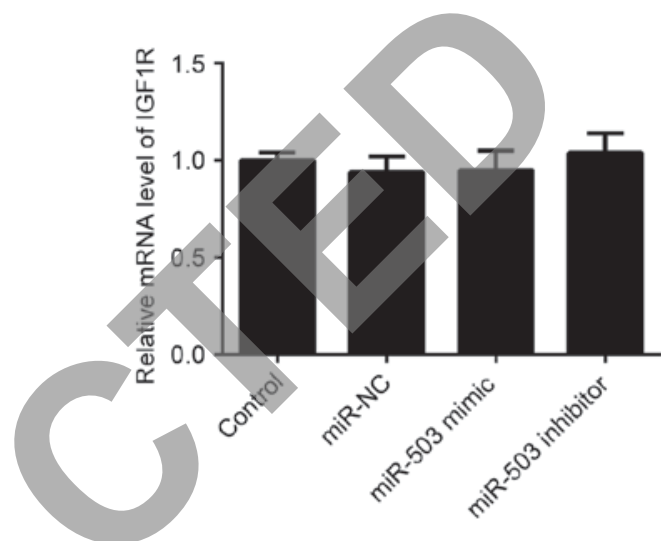

Figure 3. IGF-1R mRNA and protein expression levels in MCF-7 breast cancer cells. RT-qPCR was performed to determine the relative expression of miR-503 in MCF-7 cells transfected with an (A) miR-503 mimic or (B) miR-503 inhibitor, compared with control and miR-NC groups. (C) Western blot analysis and (D) RT-qPCR analysis of the protein and mRNA expression levels of IGF-1R in each group, respectively. Non-transfected MCF-7 cells served as a control. Data are expressed as the mean \pm standard deviation. Data are expressed as the mean \pm standard deviation. ${ }^{* *} \mathrm{P}<0.01$ vs. Control. IGF-1R, insulin-like growth factor receptor 1; NC, negative control; miR, microRNA; RT-qPCR, reverse transcription-quantitative polymerase chain reaction.

Cell invasion assay. Transwell chambers (BD Biosciences, Franklin Lakes, NJ, USA) pre-coated with Matrigel (BD Biosciences) were used to perform the Transwell assay, evaluating cell invasion. The cell suspension $\left(2 \times 10^{5}\right.$ cells $\left./ \mathrm{ml}\right)$ was prepared in DMEM, following which $300 \mu$ l cell suspension was added into the upper chamber, and $400 \mu$ 1 DMEM supplemented with $10 \%$ FBS was added into the lower chamber. Following incubation at $37^{\circ} \mathrm{C}$ in a humidified incubator containing $5 \% \mathrm{CO}_{2}$ for $24 \mathrm{~h}$, a cotton-tipped swab was used to carefully wipe out the cells that did not invade through the membrane in the filter, and the filter was then fixed in $90 \%$ alcohol. Cells were stained with $0.1 \%$ crystal violet (Sigma-Aldrich; Merck KGaA). The number of invading cells was determined under a CX23 microscope.

Dual luciferase reporter assay. The mutant type (MUT) IGF-1R 3'UTR lacking the binding sequences of miR-503 was constructed using the Directed Mutagenesis kit (Agilent Technologies, Inc., Santa Clara, CA, USA), in accordance with the manufacturer's protocol. The wild type (WT) IGF-1R 3'UTR containing the binding sequences of miR-503 was constructed using PCR. The WT or MUT IGF-1R 3'UTR was then subcloned into the downstream of the Renilla luciferase gene in the psiCHECK-2 vector (Promega Corporation, Madison,
WI, USA). MCF-7 cells were cultured to $70 \%$ confluence, and Lipofectamine 2000 was used to co-transfect MCF-7 cells with psiCHECK-2 WT IGF-1R 3'UTR vector or psiCHECK-2 MUT IGF-1R 3'UTR, and miR-503 mimic or miR-NC, respectively. At $48 \mathrm{~h}$ post-transfection, the luciferase activity was measured using the Dual-Luciferase Reporter Assay system (Promega Corporation) on an Lmax multiwell luminometer (Molecular Devices, LLC, Sunnyvale, CA).

Statistical analysis. All data are presented as the mean \pm standard deviation of at least three samples. SPSS version 17.0 software (SPSS, Inc., Chicago, IL, USA) was used to perform statistical analysis. Differences were analyzed using Student's t-test or one-way analysis of variance followed by Tukey's post hoc test. The correlation between the miR-503 and IGF-1R mRNA expression was analyzed using Pearson correlation analysis. $\mathrm{P}<0.05$ were considered to indicate a statistically significant difference.

\section{Results}

miR-503 is significantly downregulated in breast cancer. To reveal the role of miR-503 in breast cancer, the expression level of miR-503 in breast cancer tissues and their matched adjacent 
A
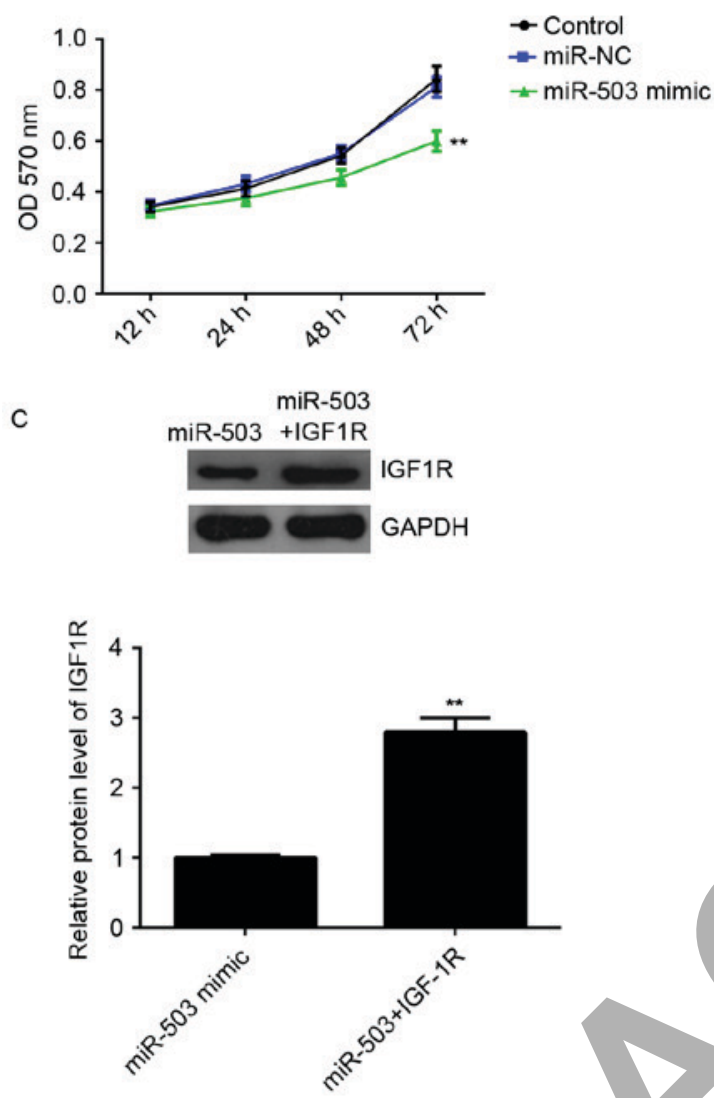

$\mathrm{E}$
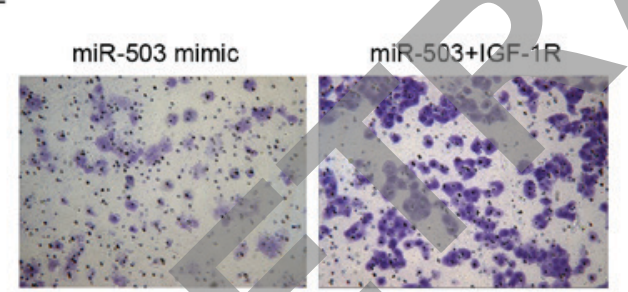
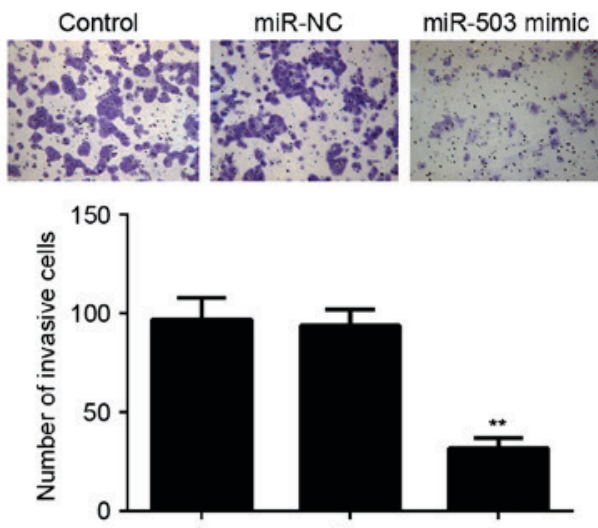

$0^{0 \times 0^{\circ}}$

(5)

sic
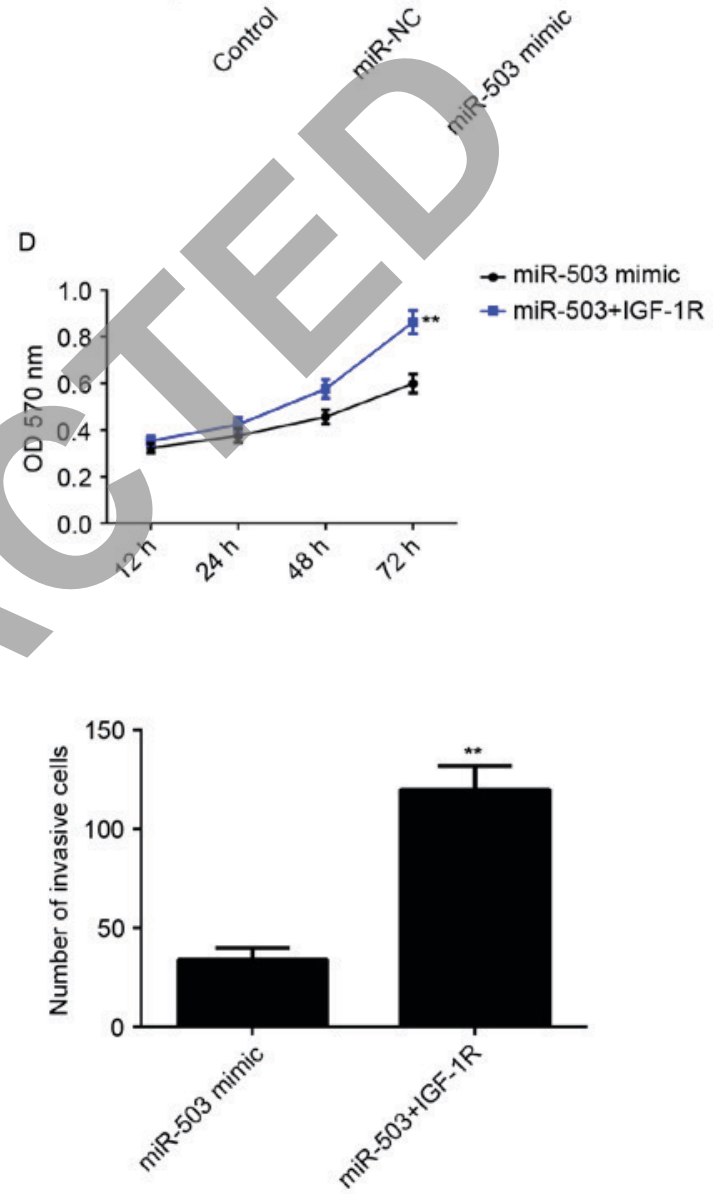

Figure 4. miR-503 inhibits the proliferation and invasion of MCF-7 cells via directly targeting IGF-1R. (A) MTT and (B) Transwell assays were used to examine cell proliferation and invasion of MCF-7 cells transfected with miR-503 mimic. Non-transfected MCF-7 cells were used as control. " $P<0.01$ vs. Control. (C) Western blot was used to examine the protein expression levels of IGF-1R in MCF-7 cells transfected with miR-503 mimic or co-transfected with miR-503 mimic and IGF-1R plasmid. (D) MTT and (E) Transwell assays were used to examine cell proliferation and invasion. ${ }^{* *} \mathrm{P}<0.01$ vs. miR-503 mimic. Data are expressed as the mean \pm standard deviation. OD, optical density; IGF-1R, insulin-like growth factor receptor 1; NC, negative control; miR, microRNA.

normal tissues was examined ( $n=23$ /group). RT-qPCR analysis demonstrated that the expression of miR-503 was significantly reduced in breast cancer tissues compared with their matched adjacent normal tissues (Fig. 1A). Furthermore, miR-503 expression levels were markedly reduced in T2-T4 stage breast cancer $(n=17)$ compared with $T 1$ stage breast cancer $(n=6$; Fig. 1B), suggesting that downregulation of miR-30-5p is involved in the malignant progression of breast cancer.

IGF-1R is a target gene of miR-503 in MCF-7 cells. The targets of miR-503 were further analyzed using bioinformatics analysis, and IGF-1R was revealed to be a putative target of miR-503 (Fig. 2A). Moreover, the predicated targeting relationship between miR-503 and IGF-1R was evolutionally conserved (Fig. 2B). To verify this predication, the luciferase reporter vectors containing the WT and MUT of IGF-1R 3'-UTR were constructed (Fig. 2C and D). The luciferase reporter assay demonstrated that the luciferase activity was significantly decreased in MCF-7 cells co-transfected with miR-503 mimic and the luciferase reporter vector containing the WT IGF-1R 3'UTR, but unaltered in MCF-7 cells co-transfected with miR-503 mimic and the luciferase reporter 
A

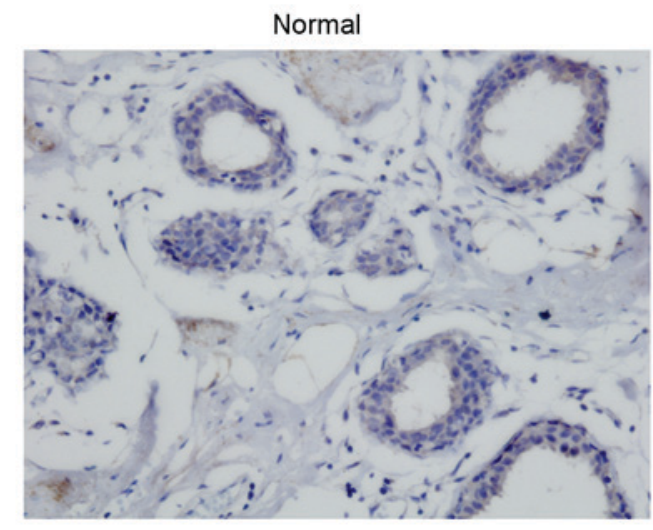

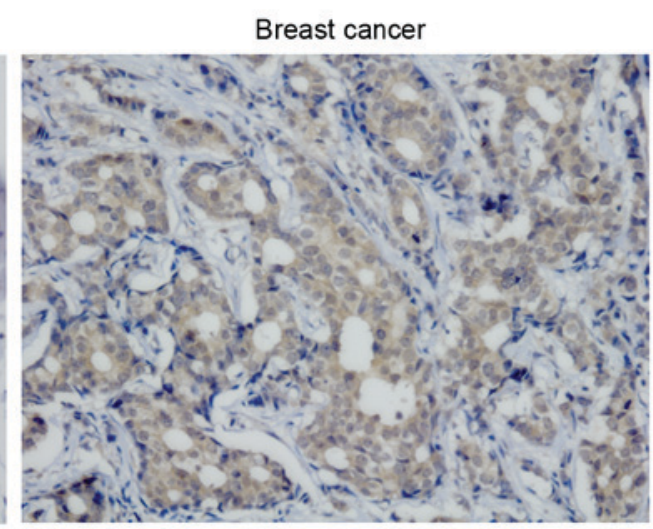

B

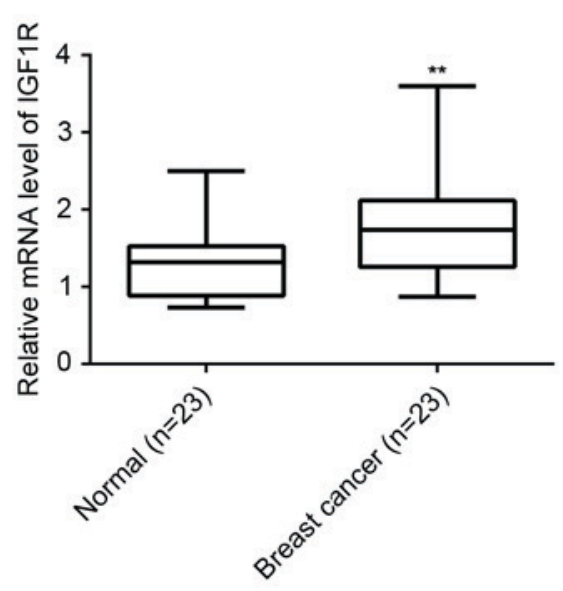

C

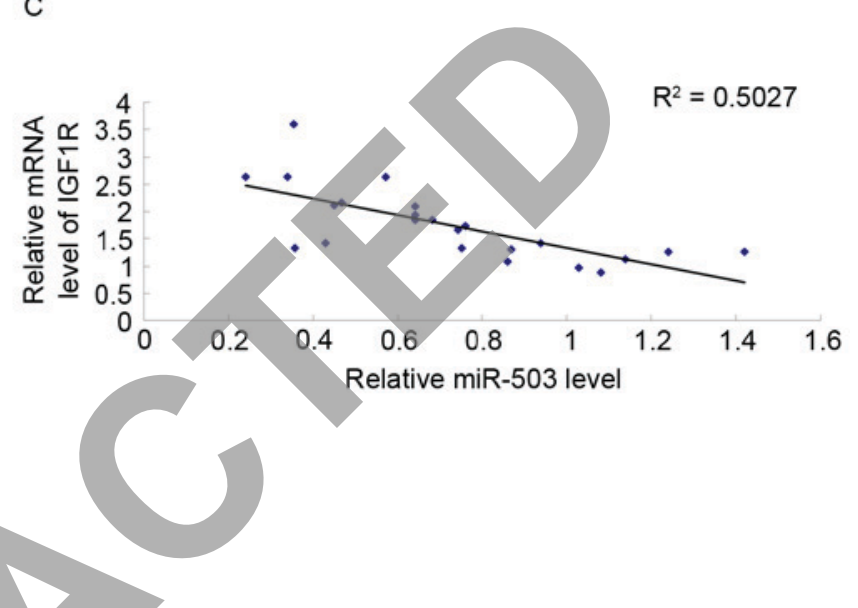

Figure 5. IGF-1R is upregulated in breast cancer and reversely correlated with miR-503 expression levels. (A) Immunohistochemical staining of protein expression levels of IGF-1R in breast cancer tissues and their matched adjacent normal tissues. Magnification, x200. (B) Reverse transcription-quantitative polymerase chain reaction was performed to examine the mRNA expression levels of IGF-1R in breast cancer tissues and their matched adjacent normal tissues. Data are expressed as the mean \pm standard deviation $\quad \mathrm{P}<0.01$ vs. Normal. (C) A reverse correlation between miR-503 and IGF-1R mRNA expression levels in breast cancer tissues. IGF-1R, insulin-like growth factor receptor 1; miR, microRNA.

vector containing the WT IGF-1R 3'UTR, compared with the control group (Fig. 2E). Therefore, IGF-1R is a direct target gene of miR-503 in MCF-7 cells. As miRs negatively regulate the expression of their target genes, the effects of miR-503 on the expression of IGF-1R in MCF-7 cells were investigated. MCF-7 cells were transfected with an miR-503 mimic or inhibitor. Following transfection, RT-qPCR was conducted to examine miR-503 levels.

Transfection with miR-503 led to a significant increase in miR-503 levels (Fig. 3A), whereas transfection with an miR-503 inhibitor markedly reduced miR-503 levels (Fig. 3B), compared with the control group. It was further observed by western blotting that overexpression of miR-30-5p significantly decreased the protein expression levels of IGF-1R, whereas knockdown of miR-503 markedly enhanced the protein expression levels of IGF-1R in MCF-7 cells, compared with the control group (Fig. 3C). However, neither miR-503 overexpression nor miR-503 inhibition affected the mRNA expression levels of IGF-1R in MCF-7 cells (Fig. 3D). Therefore, miR-503 may negatively regulate the expression of IGF-1R at the post-transcriptional level.

miR-503 inhibits the proliferation and invasion of MCF-7 cells via directly targeting IGF-1R. The role of miR-503 in the regulation of MCF-7 cell proliferation and invasion was further investigated. MTT assay was conducted to examine cell proliferation. Overexpression of miR-503 markedly inhibited MCF-7 cell proliferation compared with the control group, indicating that miR-503 may inhibit the proliferation of breast cancer cells (Fig. 4A). Furthermore, a Transwell assay was performed to examine cell invasion. As presented in Fig. 4B, overexpression of miR-503 significantly suppressed MCF-7 cell invasion compared with the control group. Therefore, miR-503 may serve a suppressive role in regulating the proliferation and invasion of breast cancer cells.

IGF-1R, an oncogene of breast cancer, was demonstrated to be a direct target of $\mathrm{miR}-503$, and its protein expression was negatively regulated by miR-503 in MCF-7 cells, it was hypothesized that IGF-1R may be involved in miR-503-mediated proliferation and invasion of MCF-7 cells. MCF-7 cells were transfected with miR-503 mimic, or co-transfected with miR-503 mimic and IGF-1R plasmid. Following transfection, the protein expression levels of IGF-1R in each group were examined, and those of IGF-1R were significantly increased in the miR-503+IGF-1R group compared with the in the miR-503 mimic group (Fig. 4C), indicating that transfection of IGF-1R plasmid reversed the suppressive effect of the miR-503 mimic on the protein expression of IGF-1R in MCF-7 cells. MTT and Transwell assays were then conducted to examine the 
cell proliferation and invasion in each group. As presented in Fig. 4D and E, the proliferation and invasion of MCF-7 cells were significantly increased in the miR-503+IGF-1R group compared with the miR-503 group, suggesting that miR-503 inhibits the proliferation and invasion of MCF-7 cells at least partially via directly targeting IGF-1R.

$I G F-1 R$ is upregulated in breast cancer and is reversely correlated with miR-503 expression levels. Finally, mRNA levels of IGF-1R in breast cancer tissues and their matched adjacent normal tissues were examined. Immunohistochemical staining and RT-qPCR indicated that the protein (Fig. 5A) and mRNA (Fig. 5B) expression levels of IGF-1R were significantly increased in breast cancer tissues compared to their matched adjacent normal tissues. Furthermore, a reverse correlation was identified between miR-503 and IGF-1R mRNA expression levels in breast cancer tissues (Fig. 5C). Taken together, these data suggested that the downregulation of miR-503 may contribute to the upregulation of IGF-1R, which promotes the malignant progression of breast cancer.

\section{Discussion}

miR-503 has been demonstrated to inhibit the proliferation of breast cancer cells. However, evidence on the underlying molecular mechanisms of miR-503 in regulating breast cancer cell proliferation and invasion is limited. The present study demonstrated that miR-503 is significantly downregulated in breast cancer tissues compared with their matched adjacent normal breast tissues. Furthermore, miR-503 expression levels were markedly reduced in T2-T4 stage breast cancer, compared with T1 stage. Further investigation suggested that miR-503 may inhibit the proliferation and invasion of MCF-7 breast cancer cells, at least partially via suppressing the protein expression levels of IGF-1R, a direct target gene of miR-503, which is significantly upregulated in breast cancer tissues.

Recently, miR-503 has been demonstrated to be involved in the development and progression of human malignancies, and serves as an oncogene or a tumor suppressor according to different cancer types. For instance, the expression levels of miR-503 are significantly increased in glioma and squamous cell carcinoma of head and neck and esophagus $(21,22)$, but decreased in anaplastic thyroid carcinomas and non-small cell lung cancer $(23,24)$. Inhibition of miR-503 expression inhibits the growth of glioma cells by targeting septin 7 and PR domain zinc finger protein 1, indicating that it serves an oncogenic role in glioma $(25,26)$. In contrast, overexpression of miR-503 inhibits cell proliferation, invasion and migration, but induces the apoptosis in hepatocellular carcinoma (HCC) cells, suggesting a tumor suppressive role of miR-503 in HCC (27). Furthermore, upregulation of miR-503 inhibits cell proliferation but induces apoptosis in colorectal cancer cells by targeting denticleless protein homolog (28). Therefore, the precise role of miR-503 is tumor-specific, and thus revealing its function in different cancer types may be important for the development of tumor-specific target therapy.

Recently, miR-503 was reported to serve a role in breast cancer. The thyroid hormone T3 was found to enhance the expression of miR-503 in breast cancer cells expressing the thyroid hormone receptor, and overexpression of miR-503 further inhibited the invasion of breast cancer cells (29). Furthermore, miR-503 was demonstrated to be downregulated in breast cancer tissues and cells, and inhibited the proliferation of breast cancer $(10,11)$. The present study also revealed a significant decrease of miR-503 expression in breast cancer tissues compared with their matched adjacent normal tissues. Additionally, its levels were markedly reduced in the T2-T4 stage of breast cancer compared with the T1 stage, suggesting that its downregulation is associated with the malignant progression of breast cancer. Furthermore, overexpression of miR-503 caused a significant decrease in MCF-7 cell proliferation and invasion, suggesting that miR-503 may have inhibitory effects on the growth and metastasis of breast cancer in vivo, which requires verification in future studies.

The present study further investigated the potential target of miR-503, which may serve as a downstream effecter involved in miR-503-mediated breast cancer cell proliferation and invasion. A luciferase reporter assay revealed that IGF-1R was a direct target gene of miR-503 in MCF-7 cells, and that the expression of IGF-1R was negatively regulated by $\mathrm{miR}-503$ at the post-transcriptional level in MCF-7 cells. IGF-1R serves an oncogenic role in tumorigenesis and cancer progression, even in the absence of the ligand and kinase activity (13). The present study demonstrated that overexpression of IGF-1R significantly reversed the suppressive effects of miR-503 on the proliferation and invasion of MCF-7 cells, suggesting that miR-503 inhibited MCF-7 cell proliferation and invasion via directly targeting IGF-1R. It has been reported that inhibition of IGF-1R effectively suppresses proliferation and induces apoptosis in breast cancer (30), and increased IGF-1R expression during neoadjuvant therapy predicts poor outcome in breast cancer patients (31). Consistent with this, the present study revealed that the mRNA and protein expression levels of IGF-1R were significantly increased in breast cancer tissues compared with their matched adjacent tissues, and its mRNA expression levels were inversely correlated with miR-503 levels in breast cancer tissues, suggesting that the downregulation of IGF-1R may contribute to the upregulation of miR-503 in breast cancer.

In conclusion, the present study demonstrated that miR-503 is significantly downregulated in breast cancer, has suppressive effects on the proliferation and invasion of breast cancer cells, at least partially via directly targeting IGF-1R, highlighting the importance of miR-503/IGF-1R axis in the malignant progression of breast cancer. These results implicate miR-503/IGF-1R as a potential therapeutic target for breast cancer.

\section{References}

1. Torre LA, Bray F, Siegel RL, Ferlay J, Lortet-Tieulent J and Jemal A: Global cancer statistics, 2012. CA Cancer J Clin 65: 87-108, 2015

2. DeSantis C, Ma J, Bryan L and Jemal A: Breast cancer statistics, 2013. CA Cancer J Clin 64: 52-62, 2014.

3. Munagala R, Aqil F, Vadhanam MV and Gupta RC: MicroRNA 'signature' during estrogen-mediated mammary carcinogenesis and its reversal by ellagic acid intervention. Cancer Lett 339: 175-184, 2013.

4. Shin VY, Siu JM, Cheuk I, Ng EK and Kwong A: Circulating cell-free miRNAs as biomarker for triple-negative breast cancer. Br J Cancer 112: 1751-1759, 2015.

5. Negrini M and Calin GA: Breast cancer metastasis: A microRNA story. Breast Cancer Res 10: 203, 2008. 
6. John B, Enright AJ, Aravin A, Tuschl T, Sander C and Marks DS: Human MicroRNA targets. PLoS Biol 2: e363, 2004.

7. Ambros V: The functions of animal microRNAs. Nature 431: 350-355, 2004

8. Bartel DP: MicroRNAs: Genomics, biogenesis, mechanism, and function. Cell 116: 281-297, 2004.

9. Lerebours F, Cizeron-Clairac G, Susini A, Vacher S, Mouret-Fourme E, Belichard C, Brain E, Alberini JL, Spyratos F, Lidereau R and Bieche I: miRNA expression profiling of inflammatory breast cancer identifies a 5-miRNA signature predictive of breast tumor aggressiveness. Int J Cancer 133: 1614-1623, 2013

10. Long J, Ou C, Xia H, Zhu Y and Liu D: MiR-503 inhibited cell proliferation of human breast cancer cells by suppressing CCND1 expression. Tumour Biol 36: 8697-8702, 2015.

11. Polioudakis D, Abell NS and Iyer VR: miR-503 represses human cell proliferation and directly targets the oncogene DDHD2 by non-canonical target pairing. BMC Genomics 16: 40, 2015.

12. King H, Aleksic T, Haluska P and Macaulay VM: Can we unlock the potential of IGF-1R inhibition in cancer therapy? Cancer Treat Rev 40: 1096-1105, 2014.

13. Crudden C, Girnita A and Girnita L: Targeting the IGF-1R: The tale of the tortoise and the hare. Front Endocrinol (Lausanne) 6 : 64, 2015.

14. Singh P, Alex JM and Bast F: Insulin receptor (IR) and insulin-like growth factor receptor 1 (IGF-1R) signaling systems: Novel treatment strategies for cancer. Med Oncol 31: 805, 2014.

15. Dricu A, Kanter L, Wang M, Nilsson G, Hjertman M, Wejde J and Larsson O: Expression of the insulin-like growth factor 1 receptor (IGF-1R) in breast cancer cells: Evidence for a regulatory role of dolichyl phosphate in the transition from an intracellular to an extracellular IGF-1 pathway. Glycobiology 9: 571-579, 1999.

16. Al Sarakbi W, Chong YM, Williams SL, Sharma AK and Mokbel K: The mRNA expression of IGF-1 and IGF-1R in human breast cancer: Association with clinico-pathological parameters. J Carcinog 5: 16, 2006.

17. Kucab JE and Dunn SE: Role of IGF-1R in mediating breast cancer invasion and metastasis. Breast Dis 17: 41-47, 2003

18. Hou X, Huang F, Macedo LF, Harrington SC, Reeves KA Greer A, Finckenstein FG, Brodie A, Gottardis MM, Carboni JM and Haluska P: Dual IGF-1R/InsR inhibitor BMS-754807 synergizes with hormonal agents in treatment of estrogen-dependent breast cancer. Cancer Res 71: 7597-7607,2011.

19. Chen Y, Zhu C, Peng Z, Dai Y and Gu Y: Lentivirus-mediated short-hairpin RNA targeting IGF-1R inhibits growth and lymphangiogenesis in breast câncer. Oncol Rep 28: 1778-1784, 2012.

20. Livak KJ and Schmittgen TD: Analysis of relative gene expression data using real-time quantitative PCR and the 2(-Delta Delta C(T)) method. Methods 25: 402-408,2001.
21. Wang K, Jia Z, Zou J, Zhang A, Wang G, Hao J, Wang Y, Yang S and Pu P: Analysis of hsa-miR-30a-5p expression in human gliomas. Pathol Oncol Res 19: 405-411, 2013.

22. Kimura S, Naganuma S, Susuki D, Hirono Y, Yamaguchi A, Fujieda S, Sano K and Itoh H: Expression of microRNAs in squamous cell carcinoma of human head and neck and the esophagus: miR-205 and miR-21 are specific markers for HNSCC and ESCC. Oncol Rep 23: 1625-1633, 2010.

23. Visone R, Pallante P, Vecchione A, Cirombella R, Ferracin M, Ferraro A, Volinia S, Coluzzi S, Leone V, Borbone E, et al: Specific microRNAs are downregulated in human thyroid anaplastic carcinomas. Oncogene 26: 7590-7595, 2007.

24. Zhu J, Zeng Y, Xu C, Qin H, Lei Z, Shen D, Liu Z and Huang JA: Expression profile analysis of microRNAs and downregulated miR-486-5p and miR-30a-5p in non-small cell lung cancer. Oncol Rep 34: 1779-1786, 2015.

25. Jia Z, Wang K, Wang G, Zhang A and Pu P: MiR-30a-5p antisense oligonucleotide suppresses glioma cell growth by targeting SEPT7. PLoS One 8: e55008, 2013.

26. Wang X, Wang K, Han L, Zhang A, Shi Z, Zhang K, Zhang H, Yang $\mathrm{S}, \mathrm{Pu} \mathrm{P}$, Shen $\mathrm{C}$, et al: PRDM1 is directly targeted by miR-30a-5p and modulates the $W n t / \beta$-catenin pathway in a Dkk1-dependent manner during glioma growth. Cancer Lett 331: 211-219, 2013

27. Dai H, Kang B, Zuo D and Zuo G: Effect of miR-30a-5p on the proliferation, apoptosis, invasion and migration of SMCC-7721 human hepatocellular carcinoma cells. Zhonghua Gan Zang Bing Za Zhi 22: 915-920, 2014 (In Chinese).

28. Baraniskin A, Birkenkamp-Demtroder K, Maghnouj A, Zöllner H, Munding J, Klein-Scory S, Reinacher-Schick A, Schwarte-Waldhoff I, Schmiegel W and Hahn SA: MiR-30a-5p suppresses tumor growth in colon carcinoma by targeting DTL. Carcinogenesis 33: 732-739, 2012.

29. Ruiz-Llorente L,Ardila-González S,FanjulLF,Martinez-Iglesias O and Aranda A: microRNAs 424 and 503 are mediators of the anti-proliferative and anti-invasive action of the thyroid hormone receptor beta. Oncotarget 5: 2918-2933, 2014.

30. Chen J, Duan Y, Zhang X, Ye Y, Ge B and Chen J: Genistein induces apoptosis by the inactivation of the IGF-1R/p-Akt signaling pathway in MCF-7 human breast cancer cells. Food Funct 6: 995-1000, 2015

31. Heskamp S, Boerman OC, Molkenboer-Kuenen JD, Wauters CA, Strobbe LJ, Mandigers CM, Bult P, Oyen WJ, van der Graaf WT and van Laarhoven HW: Upregulation of IGF-1R expression during neoadjuvant therapy predicts poor outcome in breast cancer patients. PLoS One 10: e0117745, 2015. 